\title{
IgM and IgG against Plasmodium falciparum lysate as surrogates of malaria exposure and protection during pregnancy
}

\author{
Alfredo Mayor ${ }^{1,2^{*}+}$, Carlota Dobaño ${ }^{1,2 \dagger}$, Augusto Nhabomba², Caterina Guinovart ${ }^{1,2}$, Alfons Jiménez ${ }^{1,3}$, \\ Maria Nelia Manaca ${ }^{2}$, Ruth Aguilar ${ }^{1,2,3}$, Arnoldo Barbosa ${ }^{1,2}$, Mauricio H. Rodríguez ${ }^{1,2}$, Pau Cisteró ${ }^{1}$, \\ Lazaro M. Quimice ${ }^{2}$, Clara Menéndez ${ }^{1,2}$, John J. Aponte ${ }^{1,2}$, Jaume Ordi ${ }^{1}$, Chetan E. Chitnis ${ }^{4}$ \\ and Pedro L. Alonso ${ }^{1,2}$
}

\begin{abstract}
Background: Difficulties to disentangle the protective versus exposure role of anti-malarial antibodies hamper the identification of clinically-relevant immune targets. Here, factors affecting maternal lgG and lgMs against Plasmodium falciparum antigens, as well as their relationship with parasite infection and clinical outcomes, were assessed in mothers and their children. Antibody responses among 207 Mozambican pregnant women at delivery against MSP $1_{19}$, EBA175, AMA1, DBLa and parasite lysate (3D7, R29 and E8B parasite lines), as well as the surface of infected erythrocytes, were assessed by enzyme-linked immunosorbent assay and flow cytometry. The relationship between antibody levels, maternal infection and clinical outcomes was assessed by multivariate regression analysis.

Results: Placental infection was associated with an increase in maternal levels of lgGs and IgMs against a broad range of parasite antigens. The multivariate analysis including lgGs and lgMs showed that the newborn weight increased with increasing lgG levels against a parasite lysate, whereas the opposite association was found with lgMs. IgGs are markers of protection against poor pregnancy outcomes and IgMs of parasite exposure.
\end{abstract}

Conclusions: Adjusting the analysis for the simultaneous effect of IgMs and lgGs can contribute to account for heterogeneous exposure to $P$. falciparum when assessing immune responses effective against malaria in pregnancy.

Keywords: Malaria, Pregnancy, Heterogeneity, Antibody, Immunity

\section{Background}

Plasmodium falciparum infection during pregnancy, characterized by the accumulation of parasites in the intervillous spaces of the placenta, is a major disease that affects birth outcome in sub-Saharan Africa [1], causing up to 100,000 infant deaths annually [2]. In contrast to non-pregnant adults, pregnant women are at increased risk of malaria infection independently of previous acquisition of immunity [3]. In conditions of high malaria

\footnotetext{
*Correspondence: alfredo.mayor@isglobal.org

${ }^{\dagger}$ Alfredo Mayor and Carlota Dobaño contributed equally to this work

${ }^{1}$ ISGlobal, Hospital Clínic-Universitat de Barcelona, Carrer Rosselló 153 (CEK Building), 08036 Barcelona, Spain

Full list of author information is available at the end of the article
}

transmission, this susceptibility decreases with subsequent pregnancies [3], suggesting a parity-dependent acquisition of protective immunity. Understanding the targets, quality and quantity of the immune responses that pregnant women mount upon infection and their role, in protection against infection and its adverse effects in pregnancy (i.e., low newborn weight, stillbirth and maternal anaemia) is of critical importance to design effective vaccines specific for pregnant women [4].

Immunoglobulin G (IgG) antibodies against placental infected erythrocytes (IE) and VAR2CSA [5], the major variant surface antigen on the surface of $P$. falciparum infected erythrocytes that binds chondroitin sulfate A (CSA) [6] to enable sequestration in the placenta [7], increase with parity [8]. These antibodies have been

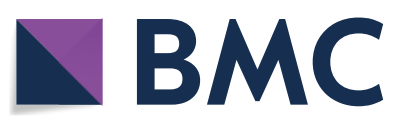

(c) The Author(s) 2018. This article is distributed under the terms of the Creative Commons Attribution 4.0 International License (http://creativecommons.org/licenses/by/4.0/), which permits unrestricted use, distribution, and reproduction in any medium, provided you give appropriate credit to the original author(s) and the source, provide a link to the Creative Commons license, and indicate if changes were made. The Creative Commons Public Domain Dedication waiver (http://creativecommons.org/ publicdomain/zero/1.0/) applies to the data made available in this article, unless otherwise stated. 
associated with reduced risk of placental infection [5, 9$11]$, low birth weight [12-15] and maternal anaemia [14, 16]. However, several other studies have failed to show such a protective association [11, 17-22], but instead suggested that antibodies at delivery reflect exposure to P. falciparum in pregnancy $[8,9,21-28]$.

Recent studies show how the power to identify immune correlates of protection in children [29-31] and pregnant women [28] under heterogeneous intensities of malaria transmission can be reduced by inclusion of individuals with different degrees of exposure [29]. Heterogeneity in exposure to $P$. falciparum $[9,22,27]$ can be taken into account by including in the analysis only women with proven parasite exposure before delivery (i.e., having had a malaria episode during pregnancy) [28]. However, this requires the morbidity surveillance at antenatal care units of rural hospitals in African countries that is not always available. There is a need to develop better methods to adjust for heterogeneity in parasite exposure when assessing immune responses that contribute to protection against malaria infection in pregnancy [30]. The aim of this study was to assess an alternative way of accounting for the effect of heterogeneous exposure to $P$. falciparum in the absence of information before delivery. Specifically, the objective was to assess the relationship of IgG and IgM against $P$. falciparum antigens in Mozambican pregnant women at delivery with parasite infection and adverse clinical impacts in the mothers and newborns. Pregnancy-specific antigens (infected erythrocytes selected from binding to CSA and placental isolates), general (non-pregnancy specific) antigens (merozoite antigens, non-CSA binder $P$. falciparum lines and isolates) and a parasite lysate (to assess responses against all whole-parasite antigens) were included. IgG but also IgM were assessed given their contribution for protection against infection [32], both in maternal and cord blood to assess the effect of placentally-transferred antibodies in reducing the risk of clinical malaria in children during their 1st year of life. The study was conducted in a mother and child cohort living in a malaria endemic area of Mozambique, in the context of a randomized, doubleblind, placebo-controlled trial ["Age of exposure and immunity to malaria in infants" (AgeMal)] [33].

\section{Methods}

\section{Study area}

The study was conducted at the Centro de Investigação em Saúde de Manhiça (CISM), located in the Manhiça District, Maputo Province, southern Mozambique. The area has been described in detail elsewhere [34]. Transmission of $P$. falciparum is perennial and of moderate intensity with a warm rainy season from November to April, and a cool dry season during the rest of the year.

\section{Study design and samples collection}

HIV-negative pregnant women resident in the Manhiça study area were recruited during the third trimester of pregnancy at the antenatal clinic of the Maragra Health Post (MHP), in the south of the study area, from September 2005 to March 2007 [33]. After delivery, neonates were evaluated for eligibility. Exclusion criteria included birth weight $<2 \mathrm{~kg}$, twins, congenital malformations, birth asphyxia or apparent health problems. Three-hundred and 49 eligible newborns were enrolled in the trial.

At delivery, maternal peripheral and cord blood samples collected into EDTA vacutainers were centrifuged and plasma stored at $-20{ }^{\circ} \mathrm{C}$ for future antibody determinations. Thin and thick smears of peripheral and cord blood were Giemsa-stained and examined for malarial parasites according to quality-control procedures [33]. Haemoglobin $(\mathrm{Hb})$ levels were determined on full blood counts performed using a Sysmex KX-21N cell counter (Sysmex Corporation, Kobe, Japan). Peripheral and cord blood was also collected onto filter papers for $P$. falciparum detection by real-time quantitative PCR (RTqPCR) in duplicates [35]. Tissue samples obtained from the maternal side of the placenta were processed for histological examination and classified as negative, acute, chronic or past infections [36]. When the delivery occurred outside the maternity post, only the mother blood sample was collected.

Children were followed up until age 24 months, with weekly active case detection from birth to age 10.5 months, and monthly home visits from 10.5 to 24 months of age. Children were examined and their parasitaemia and haematocrit determined if they presented fever (axillary temperature $\geq 37.5{ }^{\circ} \mathrm{C}$ ) or their guardians referred history of fever in the preceding $24 \mathrm{~h}$. Additionally, passive case detection was carried out at the MHP and Manhiça District Hospital (MDH) through the morbidity surveillance system to monitor attendances to the outpatient clinics and admissions to hospital.

Informed consent was sought to enroll pregnant women and their newborn children in the study upon birth. The protocol was approved by the National Mozambican Ethics Review Committee and the Hospital Clínic of Barcelona Ethics Review Committee. The trial was conducted according to the ICH Good Clinical Practice guidelines and reviewed by a Local Safety Monitor and a Data and Safety Monitoring Board. The trial was registered in ClinicalTrials.gov (clinical trials identifier NCT00231452).

Measurement of antibodies against recombinant antigens Recombinant merozoite surface protein 1 (MSP1; C-terminal 19-kD fragment, 3D7 strain) [37], erythrocytebinding antigen 175 (EBA175; region F2, Camp strain) 
[38], apical membrane antigen-1 (AMA1; full ectodomain, 3D7 strain) [39], Duffy binding-like alpha domain (DBL $\alpha$; CR1-binding minimal domain of the erythrocyte membrane protein 1 expressed by $P$. falciparum R29-rosetting + line) [40] were produced in Escherichia coli, refolded if the protein was insoluble and purified (>95\%) following previously described methods [38]. Enzyme-linked immunosorbent assay (ELISA) plates were coated with $200 \mathrm{ng}$ of recombinant antigen per well and sequentially incubated with $100 \mu \mathrm{L}$ of peripheral and cord plasma (1:500) in duplicate, and secondary peroxidase-conjugated goat anti-human IgG $(1: 30,000)$ or IgM $(1: 2000)$ [21, 41]. Reactions were developed as described previously [21] and OD values determined at $492 \mathrm{~nm}$. Fifteen plasma samples from peripheral and cord blood of Spanish pregnant women who had never been exposed to malaria were used as negative controls. The ELISA reactivity of each sample was expressed as the percentage of the value obtained with the positive pool (11 plasma samples from hyperimmune Mozambican adults) in each experiment.

Measurement of antibodies against whole parasite extract Whole-parasite lysate was prepared by three freezing/ thawing cycles of asynchronous in vitro cultures of 3D7, R29 and E8B laboratory strains at a 5\% level of parasitaemia and 1\% haematocrit, as previously described [21]. Non-infected erythrocyte (NIE) lysate, prepared using the same procedure as whole-parasite lysate, was used as a control of unspecific recognition for each plasma sample. ELISA plates were coated with $50 \mu \mathrm{L}$ of lysate per well. Wells were blocked with $300 \mu \mathrm{L}$ of $5 \%$ skim milk at $4{ }^{\circ} \mathrm{C}$ for $8 \mathrm{~h}$. One hundred microliters of plasma sample were tested in duplicate for IgG and IgM (dilution, 1: 1600). Incubation of antibodies and development of the reaction were performed as described above. Malariaspecific antibody recognition was evaluated by subtracting the mean OD value of NIEs from the mean OD value of IEs. The pool of positive plasmas was used to normalize the data from different ELISAs and results were expressed as arbitrary units. Cutoff values for positivity were calculated as the arithmetic mean plus 3 standard deviations of 14 plasma samples from Spanish pregnant women who had never been exposed to malaria.

\section{Measurement of antibodies against the surface of Plasmodium falciparum-infected erythrocytes}

Cryopreserved O+ erythrocytes infected by two placental isolates previously confirmed to express VAR2CSA [25] $\left(\mathrm{IE}_{\mathrm{Plac} 1}\right.$ and $\left.\mathrm{IE}_{\mathrm{Plac} 2}\right)$, five pediatric isolates ( $\mathrm{IE}_{\mathrm{Ch} 1-}$ $\mathrm{Ch} 4$ and $\mathrm{IE}_{\mathrm{SEV}}$ ) from Manhiça [25] and a CSA-binding parasite line adapted to culture (CS2) were thawed and matured to trophozoite stage without in vitro expansion.
Erythrocytes at $1 \%$ haematocrit were sequentially incubated with test plasma (1:20), rabbit anti-human IgG (1:200) and AlexaFluor donkey anti-rabbit IgG (1:1000) plus $10 \mu \mathrm{g} / \mathrm{mL}$ of ethidium bromide. Reactivity against the surface of IEs was expressed as the difference between the geometric mean fluorescence intensity (MFI) of 1000 IEs and the MFI of NIE obtained in a FACSCalibur flow cytometer (BD, San Jose, USA). A pool of plasma samples from 10 Mozambican pregnant women was used to normalize the data from different assays [21] and results were expressed as arbitrary units.

\section{Definitions and statistical methods}

Pregnant women were classified into first-time mothers (primigravida, PG), those with one previous pregnancy (secundigravia, SG) and those with more than two previous pregnancies (multigravida, MG). Age was categorized as $\leq 20,21-24$ and $\geq 25$ years. Maternal anaemia was considered if the haemoglobin level was $<11 \mathrm{~g} / \mathrm{dL}$ and low birth weight if the newborn weight was $<2500 \mathrm{~g}$. Distance to the river was calculated with Hawth's tools in ArcGIS software (ESRI) as the mean distance of each neighbourhood centroid to the Incomati river, and neighbourhoods were classified as those adjacent to the river $(\leq 2.5 \mathrm{~km})$ or those at a higher distance $(>2.5 \mathrm{~km})[28]$. Season during pregnancy was defined as dry if the period of pregnancy included the cool dry season (May to October), and rainy if it included four or more rainy months (November to April). Maternal malaria in peripheral blood was defined as the presence of parasites detected in peripheral blood by optical microscopy and/or RTqPCR. Placental malaria was defined by the presence of malaria parasites and/or pigment as detected by histology. Placental inflammation was defined as the presence of $>5$ mononuclear and/or polymorphonuclear leukocytes in the intervillous spaces, assessed in 10 high power fields at $400 \times[42]$.

Continuous variables were compared by ANOVA, and categorical variables by Chi squared test or Fisher's exact test. To identify factors that affect maternal IgG and IgM against $P$. falciparum antigens, the association of antibody levels (log transformed ODs or MFIs) with P. falciparum infection (maternal, placental, cord), placental inflammation, parity, neighbourhood, season and use of insecticide treated nets (ITN) or indoor residual spraying (IRS) were assessed by linear regression models. For those variables with negative values (IgGs against infected erythrocytes), prior to applying the log transform a constant value was added to the data to obtain a minimum value less than 1 . All the analyses were done univariate and multivariate, adjusting for all the other independent variables. To determine the relationship of antibody responses with clinical outcomes, parasite 
infection and clinical outcomes in mothers and their children, the association of antibody levels (independent variable) with maternal haematocrit and weight of the newborn (dependent variable) was assessed, including a multivariate analysis with all the antibody responses as independent variables. Negative binomial regression models were used to evaluate the effect of doubling the levels of antibodies on the incidence of multiple malaria episodes in children up to 12 months of age [33]. Analyses were adjusted for maternal age, parity, use of IRS, use of ITN, season, neighbourhood, child anti-malarial intervention [33] and P. falciparum infection in the mother. To assess if parity modified the associations, an interaction term was included in the regression models, and ratios and $95 \%$ confidence intervals (95\% CI) for each antibody responses were estimated after stratifying the regression models. Analyses were performed using Stata 11 (College Station, TX, USA) and significance was defined at $\mathrm{p}<0.05$.

\section{Results}

\section{Factors related to $P$. falciparum infection in pregnancy}

The analysis of antibody responses included 207 women (Table 1), 42 (20\%) with peripheral infection at delivery, $47(23 \%)$ with placental infection (acute infection in 5 [11\%], chronic infection in 1 [2\%] and past infection in $41[87 \%])$, and $8(4 \%)$ with cord blood infection. Placental inflammation in $10(5 \%)$ of the women was more frequent in women with peripheral infection than uninfected women. Placental infection decreased with parity (Table 1) and age of the women (35\% [25 out of 71] if $\leq 20$ years, $16 \%$ [ 9 out of 58 ] if $21-24$ years and $17 \%$ [ 13 out of 78 ] if $\geq 25$ years; $p=0.011$ ), and was more frequent among women living in a house that had not received IRS ( $29 \%$ [ 32 out of 109]) than in those living in a house that received IRS (15\% [15 out of 98]; $\mathrm{p}=0.020)$. Cord blood infection was more frequent among women with placental inflammation (20\% [ 2 out of 10$])$ than in those without inflammation ( $3 \%$ [ 6 out of 197]; $\mathrm{p}=0.05$ ). The use of ITN was higher among MG women (17\% [18 out of 103]) than SG (4\% [2 out of 47]) or PG women (5\% [3 out of 57]; $p=0.020$ ). LBW was more frequent among PG women than SG or MG women (Table 1).

\section{Factors related to antibody levels at delivery}

Placental infection was associated with higher levels of maternal and cord IgGs against all the antigens measured (except $\mathrm{IE}_{\mathrm{SEV}}$ ), as well as with maternal IgMs against MSP1, EBA175, AMA1 and parasite lysate (Additional file 1 Table S1). Peripheral infection (adjusted for placental infection) was associated with increased IgG levels against EBA175, parasite lysate, DBL $\alpha$ and the two placental isolates, as well as cord IgGs against DBL $\alpha$ (Additional file 1 Table S1).

Table 1 Demographic and clinical factors of mothers at delivery according to their parity

\begin{tabular}{|c|c|c|c|c|c|}
\hline & All $(n=207)$ & Primigravida $(n=57)$ & Secundigravida $(n=47)$ & Multigravida $(n=103)$ & $\mathrm{p}$ \\
\hline Placental infection, n (\%) & $47^{\mathrm{a}}(23)$ & $21(37)$ & $9(19)$ & $17(17)$ & 0.013 \\
\hline Peripheral infection, n (\%) & $42(20)$ & $14(25)$ & $7(15)$ & $21(20)$ & 0.473 \\
\hline Cord infection, n (\%) & $8(4)$ & $2(4)$ & $1(2)$ & $5(5)$ & 0.895 \\
\hline Placental density & $90.5(83.9-97.6)$ & $81.1(63.9-102.8)$ & $98.55(97.7-99.5)$ & $92.6(85.6-100.1)$ & 0.166 \\
\hline \multicolumn{6}{|l|}{ Peripheral density } \\
\hline Microscopy & $3988(894-17,778)$ & $6663(137-323,311)$ & 3652 & $3058(507-18,439)$ & 0.897 \\
\hline PCR & $0.69(0.25-1.88)$ & $1.05(0.15-7.36)$ & $0.81(0.02-33.5)$ & $0.47(0.12-2.04)$ & 0.793 \\
\hline Inflammation, n (\%) & $10(5)$ & $5(9)$ & $1(2)$ & $4(4)$ & 0.270 \\
\hline \multicolumn{6}{|l|}{ Age, mean (SD) } \\
\hline $15-20$ years & $71(34)$ & $44(77)$ & $24(51)$ & $3(3)$ & $<0.001$ \\
\hline $21-24$ years & $58(28)$ & $12(21)$ & $20(43)$ & $26(25)$ & \\
\hline$\geq 25$ years & $78(38)$ & $1(2)$ & $3(6)$ & $74(72)$ & \\
\hline Neighbourhood (<2.5km), n (\%) & $41(20)$ & $14(25)$ & $7(30)$ & $20(19)$ & 0.494 \\
\hline Use of ITN, n (\%) & $23(11)$ & $3(5)$ & $2(4)$ & $18(17)$ & 0.002 \\
\hline Household IRS, n (\%) & $98(47)$ & $24(42)$ & $21(45)$ & $53(51)$ & 0.500 \\
\hline Season (dry), n (\%) & $58(28)$ & $18(32)$ & $16(34)$ & $24(23)$ & 0.295 \\
\hline Low-birth weight, n (\%) & $28(14)$ & $15(26)$ & $6(13)$ & $7(7)$ & 0.004 \\
\hline Maternal anaemia, n (\%) & $87(42)$ & $23(40)$ & $20(43)$ & $44(43)$ & 0.792 \\
\hline
\end{tabular}

SD standard deviation, ITN insecticide-treated nets, IRS indoor residual spraying

a 41 past, 5 acute and 1 chronic infection 
Maternal IgM against MSP1, EBA175, AMA1 and DBL $\alpha$ were lower in women with placental inflammation compared to women without inflammation (Table 2). IgM $_{\mathrm{AMA} 1}$ was higher in $P$. falciparum positive cord blood (10.87, SD 9.40) than in negative cords (6.82, SD 3.75, $\mathrm{p}=0.023)$.

The use of ITNs was associated with reduced levels of maternal IgGs against DBL $\alpha$ (Additional file 1 Table S1), while IRS was associated with a reduction in the levels of maternal IgGs against lysate (Additional file 1
Table S1). Antibodies against $\mathrm{IE}_{\mathrm{ch} 3}$ were 1.32 -fold higher in neighbourhoods close to the river $(<2.5 \mathrm{~km})$ compared to those at more than $<2.5 \mathrm{~km}(95 \%$ CI $[1.02 ; 1.69]$; $\mathrm{p}=0.037$ ). There were no significant differences according to season (all p values $>0.05$ ).

Parity modified the association of maternal IgG and IgM responses as well as cord IgGs with placental infection, as indicated by the statistically significant interaction terms (Fig. 1). The analysis stratified by parity and infection showed that the increase of antibody responses

Table 2 Antibody responses in women with and without placental infection

\begin{tabular}{|c|c|c|c|c|c|c|c|c|}
\hline & \multicolumn{2}{|c|}{ No inflammation $(n=197)$} & \multicolumn{2}{|c|}{ Inflammation $(n=10)$} & \multirow[t]{2}{*}{$\mathrm{p}$} & \multicolumn{3}{|c|}{ Multivariate $^{a}$} \\
\hline & GM & SD & GM & SD & & Ratio & $(95 \% \mathrm{Cl})$ & $\mathrm{p}$ \\
\hline \multicolumn{9}{|c|}{ Maternal lgGs } \\
\hline MSP1 & 65.41 & 58.42 & 62.84 & 62.76 & 0.891 & 0.88 & $(0.49 ; 1.57)$ & 0.665 \\
\hline EBA175 & 44.17 & 33.09 & 42.24 & 26.13 & 0.853 & 0.89 & $(0.55 ; 1.44)$ & 0.636 \\
\hline AMA1 & 61.45 & 37.81 & 63.18 & 30.41 & 0.889 & 0.96 & $(0.64 ; 1.42)$ & 0.824 \\
\hline DBLa & 77.56 & 30.62 & 69.28 & 21.26 & 0.375 & 0.83 & $(0.65 ; 1.07)$ & 0.145 \\
\hline Lysate & 31.68 & 40.32 & 43.07 & 26.07 & 0.450 & 1.16 & $(0.52 ; 2.58)$ & 0.719 \\
\hline CS2 & 2.16 & 1.62 & 1.83 & 1.07 & 0.492 & 0.93 & $(0.79 ; 1.10)$ & 0.396 \\
\hline Women 1 & 149.75 & 227.78 & 93.63 & 96.67 & 0.336 & 0.68 & $(0.28 ; 1.62)$ & 0.386 \\
\hline Women2 & 412.18 & 580.46 & 216.39 & 228.31 & 0.156 & 0.56 & $(0.24 ; 1.28)$ & 0.171 \\
\hline SEV & 2.72 & 0.88 & 2.39 & 0.90 & 0.228 & 0.97 & $(0.93 ; 1.02)$ & 0.245 \\
\hline Child1 & 249.09 & 227.92 & 178.53 & 187.45 & 0.266 & 0.67 & $(0.37 ; 1.22)$ & 0.193 \\
\hline Child2 & 641.87 & 547.08 & 544.20 & 448.88 & 0.550 & 0.82 & $(0.47 ; 1.44)$ & 0.496 \\
\hline Child3 & 653.83 & 474.55 & 443.80 & 366.64 & 0.103 & 0.67 & $(0.42 ; 1.08)$ & 0.105 \\
\hline Child4 & 183.91 & 180.67 & 128.46 & 158.73 & 0.267 & 0.64 & $(0.34 ; 1.23)$ & 0.186 \\
\hline \multicolumn{9}{|c|}{ Maternal lgMs } \\
\hline MSP1 & 75.22 & 45.36 & 40.66 & 32.52 & 0.002 & 0.53 & $(0.36 ; 0.80)$ & 0.002 \\
\hline EBA175 & 39.44 & 27.08 & 18.37 & 15.24 & 0.001 & 0.46 & $(0.29 ; 0.72)$ & 0.001 \\
\hline AMA1 & 51.87 & 31.25 & 29.61 & 28.32 & 0.006 & 0.58 & $(0.38 ; 0.86)$ & 0.008 \\
\hline DBLa & 76.29 & 38.64 & 39.61 & 35.11 & $<0.001$ & 0.53 & $(0.38 ; 0.74)$ & $<0.001$ \\
\hline Lysate & 16.06 & 29.12 & 15.58 & 24.48 & 0.958 & 0.91 & $(0.28 ; 2.93)$ & 0.87 \\
\hline \multicolumn{9}{|l|}{ Cord lgGs } \\
\hline MSP1 & 54.18 & 51.79 & 41.54 & 45.09 & 0.396 & 0.69 & $(0.37 ; 1.28)$ & 0.237 \\
\hline EBA175 & 40.16 & 31.79 & 40.62 & 33.98 & 0.965 & 0.92 & $(0.55 ; 1.55)$ & 0.753 \\
\hline AMA1 & 60.89 & 40.77 & 67.26 & 27.17 & 0.643 & 1 & $(0.66 ; 1.53)$ & 0.989 \\
\hline DBLa & 60.72 & 27.78 & 65.68 & 28.77 & 0.597 & 0.98 & $(0.73 ; 1.31)$ & 0.891 \\
\hline Lysate & 28.55 & 34.11 & 25.50 & 17.50 & 0.768 & 0.77 & $(0.36 ; 1.64)$ & 0.495 \\
\hline CS2 & 2.30 & 1.73 & 2.12 & 1.39 & 0.726 & 0.98 & $(0.83 ; 1.16)$ & 0.804 \\
\hline SEV & 2.61 & 0.81 & 2.35 & 0.74 & 0.304 & 0.98 & $(0.94 ; 1.02)$ & 0.288 \\
\hline \multicolumn{9}{|l|}{ Cord IgMs } \\
\hline MSP1 & 10.76 & 5.54 & 11.92 & 8.08 & 0.546 & 1.1 & $(0.79 ; 1.54)$ & 0.575 \\
\hline EBA175 & 4.83 & 3.04 & 6.36 & 6.91 & 0.197 & 1.3 & $(0.85 ; 1.99)$ & 0.231 \\
\hline AMA1 & 6.88 & 3.79 & 8.43 & 7.34 & 0.272 & 1.23 & $(0.85 ; 1.77)$ & 0.275 \\
\hline DBLa & 10.67 & 5.45 & 12.57 & 9.02 & 0.335 & 1.18 & $(0.84 ; 1.64)$ & 0.344 \\
\hline Lysate & 6.89 & 5.64 & 6.85 & 5.77 & 0.984 & 1.06 & $(0.62 ; 1.81)$ & 0.827 \\
\hline
\end{tabular}

GM geometric mean, $S D$ standard deviation

a Adjusted for parity, age, neighbourhood, ITN, season and IRS 


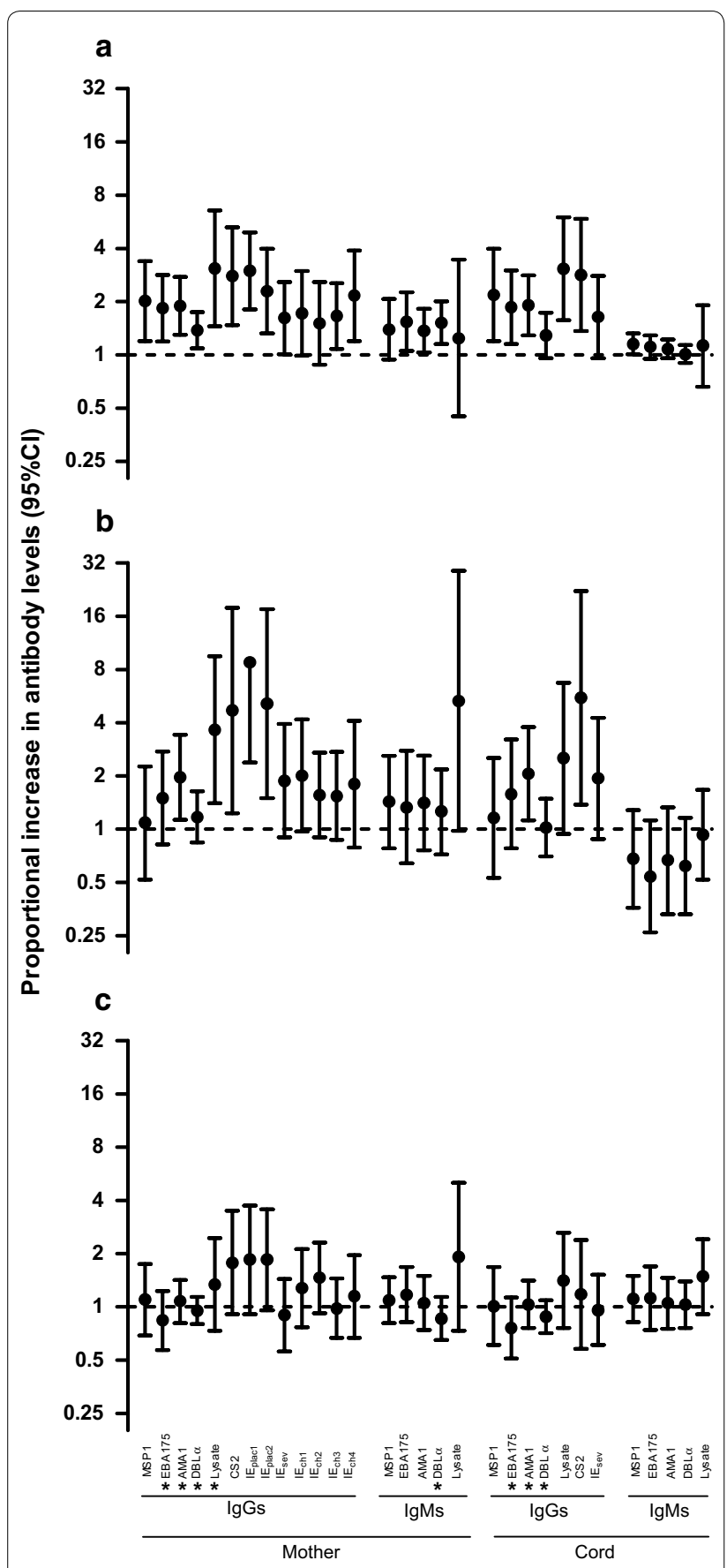

Fig. 1 Association between placental infection and antibody levels by parity. Multivariate regression analysis adjusted by peripheral infection, age, neighbourhood, household indoor residual spraying, use of insecticide treated nets and season. $Y$ axis represents the proportional increase in the level of antibodies in women with a placental infection compared to uninfected women among primigravida (a), secundigravida $(\mathbf{b})$ and multigravida $(\mathbf{c})$. *If the $p$ value for the interaction term between parity and antibody levels was $<0.05$ associated with placental infection was mainly observed in PG women (Fig. 1).

\section{Factors related to pregnancy outcome and incidence of malaria in the children}

The newborn weight increased with age and parity of the women, and was lower among women with a placental infection (Table 3). Haemoglobin levels were lower among women who had their pregnancy during the rainy season. Incidence of malaria during the first 1 year of life was higher among children born from a mother with a cord blood infection (Table 3).

The newborn weight decreased with increasing levels of maternal $\operatorname{IgM}_{\mathrm{EBA} 175}$ and $\operatorname{IgM}_{\mathrm{DBL} \alpha}$ (reductions of $0.06 \mathrm{~kg}(95 \%$ CI $[(-0.11 ;-0.01], \mathrm{p}=0.031$ and $0.07 \mathrm{~kg}$ (95\% CI $[-0.14 ;-0.01], \mathrm{p}=0.032)$ with twofold increase in antibody levels, respectively; Additional file 1: Table S2). No association was found between antibody levels and maternal anaemia. The risk of malaria in the children during their 1st year of life increased with increasing levels of maternal IgGs (all except $\operatorname{IgG}_{\mathrm{ch} 1}$ ) and cord IgGs (all except IgG-IE $\mathrm{Iev}_{\mathrm{S}}$ ). The multivariate analysis including all antibody levels showed that newborn weight increased with levels of $\operatorname{IgG}_{\text {lysate }}$ whereas decreased with levels of $\operatorname{IgM}_{\text {lysate }}$ (Fig. 2; Additional file 1: Table S3).

\section{Discussion}

This study shows that placental infection is associated with increased levels of maternal IgG and IgM against a broad range of parasite antigens and that the newborn weight decreases with increasing levels of IgM specific for EBA175 and DBL $\alpha$. No association was found between IgG levels against any of the antigens tested and improved pregnancy outcomes when the analysis was performed for specific antibodies. However, higher levels of IgG against a parasite lysate were associated with increasing birth weight when IgG and IgM were included in a multivariate analysis, being $\operatorname{IgM}$ against the parasite lysate associated with a reduction in the newborn weight. Overall, these results suggest that adjusting the analysis for the simultaneous effect of IgM and IgG could contribute to adjust for heterogeneity in parasite exposure when assessing immune responses that confer protection against malaria infection in pregnancy, being IgM markers of parasite exposure and IgG markers of protection against poor pregnancy outcomes.

Results of this study confirm parasitological observations already described for malaria in pregnancy. PG and younger women (15-20 year) were at higher risk of placental infection as detected by histology [43]. This increased risk can be attributed to a higher susceptibility to malaria during first pregnancies due to associated immune modulation [25] and/or lack of immunity 
Table 3 Factors associated with newborn weight, maternal haemoglobin levels and incidence of malaria during the 1 st year of life

\begin{tabular}{|c|c|c|c|c|c|c|c|c|c|}
\hline & \multicolumn{3}{|c|}{ Newborn weight (kg) } & \multicolumn{3}{|c|}{ Haemoglobin (g/L) } & \multicolumn{3}{|c|}{ Incidence malaria children } \\
\hline & Mean & $95 \% \mathrm{Cl}$ & $p$ & Mean & $95 \% \mathrm{Cl}$ & $\mathrm{p}$ & IRR & $95 \% \mathrm{Cl}$ & $p^{b}$ \\
\hline \multicolumn{10}{|l|}{ Parity } \\
\hline$P G$ & 2.8 & $2.69-2.91$ & $<0.001$ & 113.1 & $105.4-120.7$ & 0.878 & 1 & & \\
\hline SG & 2.9 & $2.81-2.99$ & & 113.3 & 107.9-119.6 & & 1.64 & $0.61-4.43$ & 0.331 \\
\hline MG & 3.13 & $3.05-3.20$ & & 111.5 & $107.2-115.8$ & & 1.07 & $0.44-2.59$ & 0.878 \\
\hline \multicolumn{10}{|c|}{ Placental infection } \\
\hline Negative & 3.02 & $2.95-3.08$ & 0.044 & 113 & $109.4-116.6$ & 0.473 & 1 & & \\
\hline Positive & 2.89 & $2.77-2.99$ & & 110.1 & $102.1-118.1$ & & 0.95 & $0.39-2.27$ & 0.915 \\
\hline \multicolumn{10}{|c|}{ Peripheral infection } \\
\hline Negative & 2.99 & $2.92-3.05$ & 0.990 & 113.3 & $109.6-116.9$ & 0.288 & 1 & & \\
\hline Positive & 2.99 & $2.89-3.08$ & & 108.9 & $101.1-116.7$ & & 1.82 & $0.79-4.18$ & 0.161 \\
\hline \multicolumn{10}{|c|}{ Inflammation } \\
\hline Negative & 2.98 & $2.92-3.03$ & 0.106 & 112.6 & $109.3-116.0$ & 0.488 & 1 & & \\
\hline Positive & 3.19 & $2.92-3.46$ & & 107.0 & $90.26-123.7$ & & 2.96 & $0.72-12.02$ & 0.130 \\
\hline \multicolumn{10}{|c|}{ Cord infection } \\
\hline Negative & 2.98 & $2.92-3.03$ & 0.131 & 112.6 & $109.3-115.9$ & 0.474 & 1 & & \\
\hline Positive & 3.2 & $2.72-3.68$ & & 106.4 & $88.9-123.9$ & & 5.90 & $1.53-22.77$ & $0.010^{a}$ \\
\hline \multicolumn{10}{|l|}{ Age (years) } \\
\hline $15-20$ & 2.78 & $2.69-2.87$ & $<0.001^{\mathrm{a}}$ & 110.7 & $104.7-116.8$ & 0.344 & 1 & & \\
\hline $21-24$ & 3.03 & $2.95-3.12$ & & 116.2 & $110.0-122.3$ & & 0.79 & $0.31-1.98$ & 0.608 \\
\hline$\geq 25$ & 3.14 & $3.05-3.23$ & & 110.9 & $105.7-116.0$ & & 0.88 & $0.38-2.05$ & 0.769 \\
\hline \multicolumn{10}{|c|}{ Neighbourhood (km) } \\
\hline$>2.5$ & 3.00 & $2.94-3.06$ & 0.328 & 111.1 & $114.7-107.5$ & 0.122 & 1 & & \\
\hline$<2.5$ & 2.93 & $2.79-3.07$ & & 117.6 & $109.9-125.2$ & & 1.04 & $0.42-2.57$ & 0.936 \\
\hline \multicolumn{10}{|l|}{ Use of ITN } \\
\hline Negative & 2.97 & $2.91-3.03$ & 0.171 & 112.8 & $109.2-116.5$ & 0.469 & 1 & & \\
\hline Positive & 3.09 & $2.92-3.28$ & & 109.1 & $102.4-115.9$ & & 1.07 & $0.34-3.35$ & 0.903 \\
\hline \multicolumn{10}{|l|}{ Use of IRS } \\
\hline Negative & 2.94 & $2.87-3.02$ & 0.109 & 113.3 & $108.5-118.1$ & 0.573 & 1 & & \\
\hline Positive & 3.04 & $2.95-3.12$ & & 111.4 & $106.8-115.9$ & & 0.66 & $0.32-1.37$ & 0.268 \\
\hline \multicolumn{10}{|l|}{ Season } \\
\hline Rainy & 2.98 & $2.91-3.05$ & 0.685 & 110.2 & $106.2-114.3$ & $0.049^{a}$ & 1 & & \\
\hline Dry & 3.01 & $2.89-3.11$ & & 117.4 & $111.8-123.0$ & & 0.99 & $0.44-2.21$ & 0.980 \\
\hline
\end{tabular}

$P G$ primigravida, $S G$ secundigravida, $M G$ multigravida, ITN insecticide-treated nets, IRS indoor residual spraying, IRR incidence rate ratio

${ }^{a} \mathrm{p}<0.05$ in the multivariate analysis adjusted by age, use of IRS and ITS, season and neighbourhood

b adjusted for treatment, weight and maternal haemoglobin

against VAR2CSA expressed by placental parasites [5]. Differential use of preventive tools among women at different parities, as shown by the higher use of ITN among MG women compared to PG, may also contribute to reduce the risk of malaria infection in MG women. In accordance with this reduction of placental infection with increasing parity, low birth weight was more common in PG and the newborn weight was reduced in mothers with placental infection and younger women. Importantly, household IRS was also associated with a reduction in the prevalence of placental infection. In contrast to placental infection, peripheral infection was not found to be associated with parity, possibly because placental submicroscopic infections were not assessed in this study. Results of this study also show that incidence of malaria was higher among children born from women living closer to the river, an association that can be explained by similar risk of exposure to the parasites in mothers and infants living in the same household [21]. Furthermore, this study shows that cord blood infections 

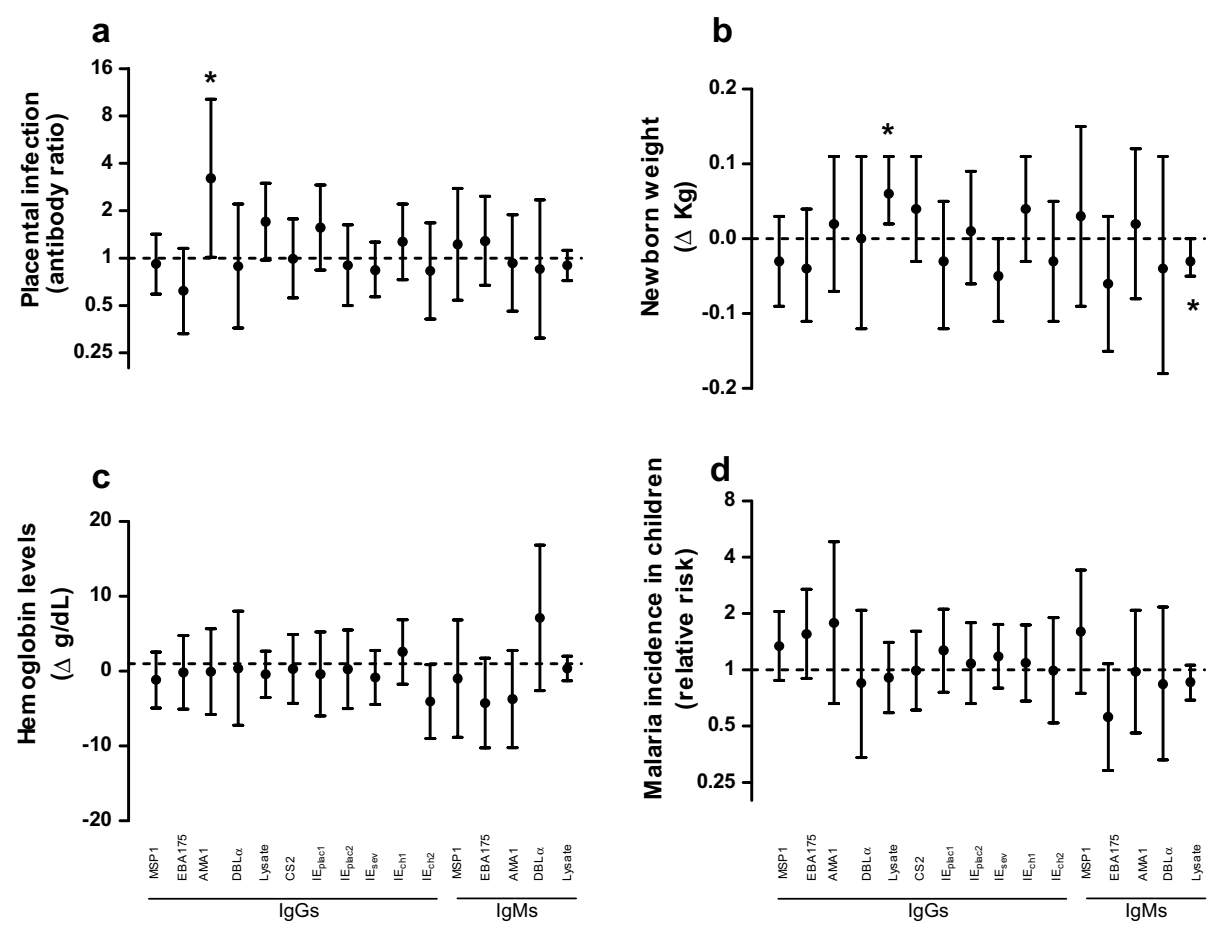

Fig. 2 Association of antibody levels with maternal infection, pregnancy outcomes and incidence of malaria in children. Multivariate analysis including all the antibodies and adjusted for parity, age, neighbourhood, season, household indoor residual spraying and use of insecticide treated nets. Y axis represent the adjusted ratio of antibody levels between women with placental infection and women without infection (a), the effect of a twofold increase in antibody levels on the newborn weight (b), on maternal haemoglobin levels (c) and on the risk of malaria episodes in infants (d). $\triangle$ Expresses the increase or decrease of newborn weight or maternal haemoglobin levels with twofold increases in antibody levels. ${ }^{*}$ identifies statistically significant $p$ values $(<0.05)$

are associated with an increased risk of malaria during the 1st year of life, suggesting that in utero exposure can have a detrimental effect on the ability of newborns to mount an effective immune response against the parasite.

In this maternal cohort, placental infection is associated with an increase of IgG, as already shown in studies conducted in the same study area [21, 28], but also with increasing IgM. The magnitude of this increase was higher in PG than in MG, probably because parasite densities are higher in first time pregnant women, leading to a higher boosting of antibodies. Antibody responses were associated with a non-significant reduction of haemoglobin levels in the women at delivery, and the multivariate analysis showed that IgM against the lysate were associated with a reduction in the newborn weight. Although the large number of comparisons made may have increased the chance of false discoveries, the fact that this associations with antibody responses against parasite lysate are maintained in the multivariate analysis including all the antigens suggest the genuineness of the observation. Moreover, antibody responses were associated with an increase in the incidence of malaria in their children during their 1st year of life. This association may be explained by a similarly high exposure to mosquito bites in pregnant women and their children residing in the same household which would translate into a boosting of antibody responses in pregnant women at delivery and a higher malaria incidence in the children. Overall, these results reinforce the concept of antibodies as markers of parasite exposure and the difficulties of disentangling the protective versus exposure role of antibodies in a situation of heterogeneous exposure. In absence of a continuous morbidity surveillance at antenatal care units to identify malaria cases and exclude from the analysis those women without proven exposure during pregnancy (i.e., having had a malaria episode), these results suggest that adjusting for IgM as markers of exposure to the parasite can control for the heterogeneity of exposure [28] and contribute to identify those IgG that may have a role in protection. Interestingly, data of this study show that IgG against a parasite lysate are more associated to an increase in the newborn weight than IgG against the surface of a CSA-binding parasite line. This association might be explained by an antibody-mediated clearance of circulating parasites early at pregnancy (i.e., before these parasite populations switch to placental-binding, that 
can contribute to prevent low birthweight. Also, immunity against a broad range of $P$. falciparum antigens and not only against CS2 may have a role in controlling parasite infections and reducing the adverse consequences of malaria in pregnancy.

\section{Conclusions}

Newborn weight increased with increasing IgG levels against a parasite lysate, whereas the opposite association was found with IgM, suggesting that IgG are markers of protection against poor pregnancy outcomes and IgM of parasite exposure. This study confirms the need for developing methods to control for heterogeneous exposure to the parasite when aiming to identify those immune responses that have a potential role in protection against infection and disease. Further studies are needed to assess novel approaches to disentangle exposure from protection in immunological studies assessing the role of antibodies against the adverse consequences of malaria infection in situations of heterogeneous malaria exposure.

\section{Additional file}

Additional file 1: Table S1. Association of antibody responses with maternal infection, use of insecticide treated nets and indoor residual spraying. Table S2. Association between antibody levels and weight of newborn, maternal hemoglobin and incidence of malaria in children. Table S3. Association of antibody levels with maternal infection, pregnancy outcomes and incidence of malaria in children.

\section{Abbreviations}

MSP1: merozoite surface protein 1; EBA175: erythrocyte-binding antigen 175; AMA1: apical membrane antigen-1; DBLa: Duffy binding-like alpha domain; ELISA: enzyme-linked immunosorbent assay; lgG: immunoglobulin G; IgM: immunoglobulin M; IE: infected erythrocytes; CSA: chondroitin sulfate A; RTqPCR: real-time quantitative PCR; Hb: haemoglobin; NIE: non-infected erythrocyte; MFI: mean fluorescence intensity; CISM: Centro de Investigação em Saúde de Manhiça; MDH: Manhiça District Hospital; MHP: Maragra Health Post; HIV: human immunodeficiency virus; PG: primigravida; SG: secundigravida; MG: multigravida; ITN: insecticide-treated nets; IRS: indoor residual spraying.

\section{Authors' contributions}

AM, CD, JJA, PLS designed the study. CG coordinated the field clinical epidemiological study. MNM, AN, RA, AB, MHR, LMQ processed the samples. LMQ, JO performed histological examination placentas. MNM, AJ, PC, CEC performed molecular and immunological laboratory analyses. AM, CD analyzed and interpreted the data. AM wrote the first draft. AN, CG, RA, JO, CM, CD contributed in writing the manuscript. All authors read and approved the final manuscript.

\section{Author details}

${ }^{1}$ ISGlobal, Hospital Clínic-Universitat de Barcelona, Carrer Rosselló 153 (CEK Building), 08036 Barcelona, Spain. ${ }^{2}$ Centro de Investigação em Saúde de Manhiça (CISM), Maputo, Mozambique. ${ }^{3}$ CIBER Epidemiología y Salud Pública (CIBERESP), Madrid, Spain. ${ }^{4}$ International Centre for Genetic Engineering and Biotechnology, New Delhi, India.

\section{Acknowledgements}

We thank all children and their families for their participation in the study; the field workers, field supervisors, laboratory staff and other staff at CISM for their work during the study; Laura Puyol for laboratory support; Llorenç Quintó for statistical analyses support; Sònia Tomàs for her work as project manager.

\section{Competing interests}

The authors declare that there are no competing interests.

\section{Availability of data and materials}

The datasets used and/or analyzed during the current study are available from the corresponding author on reasonable request.

\section{Ethics approval and consent to participate}

The protocol was approved by the National Mozambican Ethics Review Committee and the Hospital Clinic of Barcelona Ethics Review Committee. Written informed consent was obtained to enroll mothers and their infants.

\section{Funding}

The study was funded by a EU Framework Program 6 STREP project (Malaria age exposure, Project reference 18902), the Spanish Ministerio de Ciencia e Innovación (SAF2005-25642-E, salary support RYC-2008-02631 for CD) and the Instituto de Salud Carlos III (A107190024, salary support CM04/00028 and CES10/021-I3SNS to CG and AM, respectively). AM received support from the Departament d'Universitats i Recerca de la Generalitat de Catalunya [Agencia de Gestión de Ayudas Universitarias y de Investigación (AGAUR; grant 2014SGR263). The Manhiça Health Research Centre receives core funding from the Spanish Agency for International Cooperation and Development (AECID). ISGlobal is a member of the CERCA Programme, Generalitat de Catalunya.

\section{Publisher's Note}

Springer Nature remains neutral with regard to jurisdictional claims in published maps and institutional affiliations.

Received: 24 January 2018 Accepted: 24 April 2018

Published online: 10 May 2018

\section{References}

1. Menendez C, Ordi J, Ismail MR, Ventura PJ, Aponte JJ, Kahigwa E, et al. The impact of placental malaria on gestational age and birth weight. J Infect Dis. 2000;181:1740-5.

2. Desai M, ter Kuile FO, Nosten F, McGready R, Asamoa K, Brabin B, et al. Epidemiology and burden of malaria in pregnancy. Lancet Infect Dis. 2007;7:93-104.

3. Brabin BJ. An analysis of malaria in pregnancy in Africa. Bull World Health Organ. 1983;61:1005-6.

4. Fried M, Duffy PE. Malaria during pregnancy. Cold Spring Harb Perspect Med. 2017:7:a025551.

5. Fried M, Nosten F, Brockman A, Brabin BJ, Duffy PE. Maternal antibodies block malaria. Nature. 1998:395:851-2.

6. Fried M, Duffy PE. Adherence of Plasmodium falciparum to chondroitin sulfate A in the human placenta. Science. 1996;272:1502-4.

7. Salanti A, Staalsoe T, Lavstsen T, Jensen AT, Sowa MP, Arnot DE, et al. Selective upregulation of a single distinctly structured var gene in chondroitin sulphate A-adhering Plasmodium falciparum involved in pregnancyassociated malaria. Mol Microbiol. 2003;49:179-91.

8. Oleinikov AV, Rossnagle E, Francis S, Mutabingwa TK, Fried M, Duffy PE. Effects of sex, parity, and sequence variation on seroreactivity to candidate pregnancy malaria vaccine antigens. J Infect Dis. 2007;196:155-64.

9. Tutterrow YL, Avril M, Singh K, Long CA, Leke RJ, Sama G, et al. High levels of antibodies to multiple domains and strains of VAR2CSA correlate with the absence of placental malaria in Cameroonian women living in an area of high Plasmodium falciparum transmission. Infect Immun. 2012;80:1479-90

10. Guitard J, Cottrell G, Magnouha NM, Salanti A, Li T, Sow S, et al. Differential evolution of anti-VAR2CSA- IgG3 in primigravidae and multigravidae pregnant women infected by Plasmodium falciparum. Malar J. 2008;7:10.

11. Tuikue Ndam NG, Salanti A, Le-Hesran JY, Cottrell G, Fievet N, Turner L, et al. Dynamics of anti-VAR2CSA immunoglobulin $\mathrm{G}$ response in a cohort of senegalese pregnant women. J Infect Dis. 2006;193:713-20.

12. Duffy PE, Fried M. Antibodies that inhibit Plasmodium falciparum adhesion to chondroitin sulfate $A$ are associated with increased birth weight and the gestational age of newborns. Infect Immun. 2003;71:6620-3. 
13. Salanti A, Dahlback M, Turner L, Nielsen MA, Barfod L, Magistrado P, et al. Evidence for the involvement of VAR2CSA in pregnancy-associated malaria. J Exp Med. 2004;200:1197-203.

14. Staalsoe T, Shulman CE, Bulmer JN, Kawuondo K, Marsh K, Hviid L. Variant surface antigen-specific $\lg G$ and protection against clinical consequences of pregnancy-associated Plasmodium falciparum malaria. Lancet. 2004;363:283-9.

15. Ataide R, Mwapasa V, Molyneux ME, Meshnick SR, Rogerson SJ. Antibodies that induce phagocytosis of malaria infected erythrocytes: effect of HIV infection and correlation with clinical outcomes. PLOS ONE. 2011;6:e22491.

16. Feng G, Aitken E, Yosaatmadja F, Kalilani L, Meshnick SR, Jaworowski A, et al. Antibodies to variant surface antigens of Plasmodium falciparuminfected erythrocytes are associated with protection from treatment failure and the development of anemia in pregnancy. J Infect Dis. 2009;200:299-306.

17. Aitken EH, Mbewe B, Luntamo M, Maleta K, Kulmala T, Friso MJ, et al. Antibodies to chondroitin sulfate A-binding infected erythrocytes: dynamics and protection during pregnancy in women receiving intermittent preventive treatment. J Infect Dis. 2010:201:1316-25.

18. Ataide R, Hasang W, Wilson DW, Beeson JG, Mwapasa V, Molyneux ME, et al. Using an improved phagocytosis assay to evaluate the effect of HIV on specific antibodies to pregnancy-associated malaria. PLoS ONE. 2010;5:e10807.

19. Cox SE, Staalsoe T, Arthur P, Bulmer JN, Hviid L, Yeboah-Antwi K, et al. Rapid acquisition of isolate-specific antibodies to chondroitin sulfate A-adherent plasmodium falciparum isolates in Ghanaian primigravidae. Infect Immun. 2005;73:2841-7.

20. Fievet N, Le Hesran JY, Cottrell G, Doucoure S, Diouf I, Ndiaye JL, et al. Acquisition of antibodies to variant antigens on the surface of Plasmodium falciparum-infected erythrocytes during pregnancy. Infect Genet Evol. 2006;6:459-63.

21. Serra-Casas E, Menendez C, Bardaji A, Quinto L, Dobano C, Sigauque B, et al. The effect of intermittent preventive treatment during pregnancy on malarial antibodies depends on HIV status and is not associated with poor delivery outcomes. J Infect Dis. 2009;201:123-31.

22. Megnekou R, Staalsoe T, Taylor DW, Leke R, Hviid L. Effects of pregnancy and intensity of Plasmodium falciparum transmission on immunoglobulin $\mathrm{G}$ subclass responses to variant surface antigens. Infect Immun. 2005:73:4112-8.

23. Beeson JG, Mann EJ, Elliott SR, Lema VM, Tadesse E, Molyneux ME, et al. Antibodies to variant surface antigens of Plasmodium falciparum-infected erythrocytes and adhesion inhibitory antibodies are associated with placental malaria and have overlapping and distinct targets. J Infect Dis. 2004;189:540-51.

24. Maubert B, Fievet N, Tami G, Cot M, Boudin C, Deloron P. Development of antibodies against chondroitin sulfate A-adherent Plasmodium falciparum in pregnant women. Infect Immun. 1999;67:5367-71.

25. Mayor A, Rovira-Vallbona E, Machevo S, Bassat Q, Aguilar R, Quinto L, et al. Parity and placental infection affect antibody responses against Plasmodium falciparum during pregnancy. Infect Immun. 2011;79:1654-9.

26. Okoko BJ, Wesumperuma LH, Ota MO, Pinder M, Banya W, Gomez SF, et al. The influence of placental malaria infection and maternal hypergammaglobulinemia on transplacental transfer of antibodies and lgG subclasses in a rural West African population. J Infect Dis. 2001;184:627-32.

27. Staalsoe T, Megnekou R, Fievet N, Ricke CH, Zornig HD, Leke R, et al. Acquisition and decay of antibodies to pregnancy-associated variant antigens on the surface of Plasmodium falciparum-infected erythrocytes that protect against placental parasitemia. J Infect Dis. 2001;184:618-26.

28. Mayor A, Kumar U, Bardaji A, Gupta P, Jimenez A, Hamad A, et al. Improved pregnancy outcomes in women exposed to malaria with high antibody levels against Plasmodium falciparum. J Infect Dis. 2013;207:1664-74.
29. Greenhouse B, Ho B, Hubbard A, Njama-Meya D, Narum DL, Lanar DE, et al. Antibodies to Plasmodium falciparum antigens predict a higher risk of malaria but protection from symptoms once parasitemic. J Infect Dis. 2011;204:19-26.

30. Bousema T, Kreuels B, Gosling R. Adjusting for heterogeneity of malaria transmission in longitudinal studies. J Infect Dis. 2011;204:1-3.

31. Kinyanjui SM, Bejon P, Osier FH, Bull PC, Marsh K. What you see is not what you get: implications of the brevity of antibody responses to malaria antigens and transmission heterogeneity in longitudinal studies of malaria immunity. Malar J. 2009;8:242.

32. Pleass RJ, Moore SC, Stevenson L, Hviid L. Immunoglobulin M: restrainer of inflammation and mediator of immune evasion by Plasmodium falciparum malaria. Trends Parasitol. 2016;32:108-19.

33. Guinovart C, Dobano C, Bassat Q, Nhabomba A, Quinto L, Manaca MN, et al. The role of age and exposure to Plasmodium falciparum in the rate of acquisition of naturally acquired immunity: a randomized controlled trial. PLoS ONE. 2012;7:e32362.

34. Alonso P, Saute F, Aponte JJ, Gomez-Olive FX, Nhacolo A, Thomson R, et al. Manhica DSS, Mozambique. Population, Health and Survival at INDEPTH Sites. 2001;1:189-95.

35. Mayor A, Serra-Casas E, Bardaji A, Sanz S, Puyol L, Cistero P, et al. Submicroscopic infections and long-term recrudescence of Plasmodium falciparum in Mozambican pregnant women. Malar J. 2009;8:9.

36. Ordi J, Ismail MR, Ventura PJ, Kahigwa E, Hirt R, Cardesa A, et al. Massive chronic intervillositis of the placenta associated with malaria infection. Am J Surg Pathol. 1998;22:1006-11.

37. Mazumdar S, Sachdeva S, Chauhan VS, Yazdani SS. Identification of cultivation condition to produce correctly folded form of a malaria vaccine based on Plasmodium falciparum merozoite surface protein-1 in Escherichia coli. Bioprocess Biosyst Eng. 2010;33:719-30.

38. Pandey KC, Singh S, Pattnaik P, Pillai CR, Pillai U, Lynn A, et al. Bacterially expressed and refolded receptor binding domain of Plasmodium falciparum EBA-175 elicits invasion inhibitory antibodies. Mol Biochem Parasitol. 2002;123:23-33.

39. Kocken $\mathrm{CH}$, Withers-Martinez $\mathrm{C}$, Dubbeld MA, van der Wel A, Hackett $F$, Valderrama A, et al. High-level expression of the malaria blood-stage vaccine candidate Plasmodium falciparum apical membrane antigen 1 and induction of antibodies that inhibit erythrocyte invasion. Infect Immun. 2002;70:4471-6.

40. Mayor A, Rovira-Vallbona E, Srivastava A, Sharma SK, Pati SS, Puyol L, et al. Functional and immunological characterization of a Duffy binding-like alpha domain from Plasmodium falciparum erythrocyte membrane protein 1 that mediates rosetting. Infect Immun. 2009;77:3857-63.

41. Naniche D, Serra-Casas E, Bardaji A, Quinto L, Dobano C, Sigauque B, et al. Reduction of antimalarial antibodies by HIV infection is associated with increased risk of Plasmodium falciparum cord blood infection. J Infect Dis. 2012;205:568-77.

42. Ismail MR, Ordi J, Menendez C, Ventura PJ, Aponte JJ, Kahigwa E, et al. Placental pathology in malaria: a histological, immunohistochemical, and quantitative study. Hum Pathol. 2000;31:85-93.

43. Brabin BJ, Romagosa C, Abdelgalil S, Menendez C, Verhoeff FH, McGready $\mathrm{R}$, et al. The sick placenta-the role of malaria. Placenta. 2004;25:359-78.

Ready to submit your research? Choose BMC and benefit from:

- fast, convenient online submission

- thorough peer review by experienced researchers in your field

- rapid publication on acceptance

- support for research data, including large and complex data types

- gold Open Access which fosters wider collaboration and increased citations

- maximum visibility for your research: over $100 \mathrm{M}$ website views per year

At $\mathrm{BMC}$, research is always in progress.

Learn more biomedcentral.com/submissions 